\title{
Targeted next-generation sequencing identification of a novel missense mutation of the SKIV2L gene in a patient with trichohepatoenteric syndrome
}

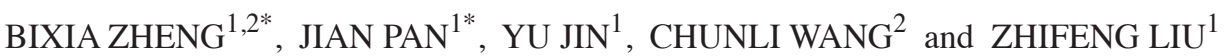 \\ ${ }^{1}$ Department of Gastroenterology; ${ }^{2}$ Nanjing Key Laboratory of Pediatrics, \\ Nanjing Children's Hospital Affiliated to Nanjing Medical University, \\ Nanjing, Jiangsu 210008, P.R. China
}

Received July 27, 2015; Accepted June 1, 2016

DOI: $10.3892 / \mathrm{mmr} .2016 .5503$

\begin{abstract}
Trichohepatoenteric syndrome (THES) is a rare autosomal, recessively inherited disorder. Mutations in the tetratricopeptide repeat domain 37 (TTC37) gene and the superkiller viralicidic activity 2-like (SKIV2L) gene have been identified to cause THES. The present study reported a case of a Chinese boy, who presented clinically with intrauterine growth retardation, intractable diarrhea, facial dysmorphism, abnormal scalp hair shafts, immune disorders and liver involvement. Targeted next-generation sequencing and Sanger DNA sequencing showed compound heterozygous mutations of the SKIV2L gene. The present study was the first, to the best of our knowledge, to report a case of a boy with THES resulting from compound heterozygous mutations of the SKIV2L gene in China. Target sequence capture combined with high-throughput next-generation sequencing technologies have shown to be effective methods for the molecular genetic assessment of rare inherited disorders.
\end{abstract}

\section{Introduction}

Trichohepatoenteric syndrome (THES; MIM 222470), also termed syndromic or phenotypic diarrhea, is an autosomal, recessively inherited disorder with an estimated incidence of 1/300,000-400,000 live births (1-5). This syndrome was first recognized in 1982, with a report on two siblings, who were described as having unexplained diarrhea and failure to thrive,

Correspondence to: Dr Zhifeng Liu, Department of Gastroenterology, Nanjing Children's Hospital Affiliated to Nanjing Medical University, 72 Guangzhou Road, Nanjing, Jiangsu 210008, P.R. China

E-mail: liuzfnj@hotmail.com

${ }^{*}$ Contributed equally

Key words: trichohepatoenteric syndrome, superkiller viralicidic activity 2-like gene, next-generation sequencing with facial dysmorphism and abnormal scalp hair shafts (6). This syndrome was initially described by Verloes et al (7) in 1997 as a different syndrome. THES is characterized by intractable diarrhea, facial dysmorphism, intrauterine growth retardation, immunodeficiency and hair abnormalities. Hepatic involvement varies from no abnormality to cirrhosis and hemochromatosis $(2,5,8)$. Previously, mutations in the tetratricopeptide repeat domain 37 (TTC37) gene and the superkiller viralicidic activity 2-like (SKIV2L) gene have been identified to cause THES $(9,10)$. In 2010, the TTC37 gene on chromosome 5 was identified as the causative gene for THES (4). Genetic heterogeneity in THES has previously been documented, with causative mutations also identified in the $S K I V 2 L$ gene (9).

The present study reported the case of a Chinese boy with THES resulting from compound heterozygous mutations of the SKIV2L gene. Genetic analysis of his family was also performed, and his parents appeared to be healthy, but carried heterozygous mutations. The findings in the present study, which are associated with phenotype and the new point mutation in the SKIV2L gene, will increase our understanding of the characteristics of THES in the Chinese population.

\section{Materials and methods}

Peripheral blood collection and preparation. At the age of 17 months, the proband was hospitalized in our department due to intractable diarrhea, severe protein energy malnutrition and failure to thrive. During this hospitalization, peripheral blood samples were collected from the proband and his parents. A 2-4 ml volume of peripheral blood was collected in disposable vacuum tubes for genetic analysis. Genomic DNA was isolated from peripheral leukocytes under standard methods using a DNA isolation kit (Tiangen, China), according to the manufacturer's protocol The study protocol was approved by the ethics committee of the Children's Hospital of Nanjing Medical University (Nanjing, China). Written informed consent was obtained from the proband and his parents for publication of the case report and any accompanying images.

Targeted sequence capture and next-generation sequencing. The genomic DNA sample from the proband was sheared by 
sonication. The sheared genomic DNA was then hybridized using a NimbleGen probe capture array (Joy Orient Translational Medicine Research Center Co., Ltd., Beijing, China). The array covered 200 genes from the Online Mendelian Inheritance in Man database (http://www.omim.org), which are associated with diarrhea. The libraries were first assessed for enrichment using quantitative polymerase chain reaction (PCR) analysis, and for size distribution and concentration using an Agilent Bioanalyzer 2100 (Agilent Technologies, Inc., Santa Clara, CA, USA). The samples were then sequenced on an Illumina Hiseq2500 sequencing system (Illumina, Inc., San Diego, CA, USA). Two parallel reactions were performed for each sample.

Data filtering, mapping and variant detection. The raw image files were processed using BclToFastq (Illumina, Inc.) for base calling and generating the raw data. The low-quality variations were filtered out using the cut-off quality score of $\geq 20$. The sequencing reads were aligned to the National Center for Biotechnology Information (NCBI) human reference genome (hg19) using Burrows-Wheeler Alignment. Samtools (http://(samtools.sourceforge.net) and Pindel (http://www.ebi. ac.uk/ kye/pindel/) were used to analyze the single nucleotide polymophisms (SNPs) and indels of the sequence.

Mutation confirmation using Sanger sequencing. To confirm the variants identified using exome sequencing, Sanger sequencing was performed to analyze the DNA sequences, which included any nucleotide variant. DNA sequences containing the mutated base (c.1891G >A) and the flanking bases of the SKIV $2 L$ gene were amplified using PCR with following primers: Forward 5'-ATA GGGTGTTCCGAGACTCCATCC-3' and reverse 5'-CTTTCC ACCACCACATGCACCTT-3'. Another mutation (c.1120C>T) was amplified with the following primers: Forward 5'-ACT GGCTAACTTCATGCTCTCTTC-3' and reverse 5'-CCCTGT ATTGAGTGTCCATCTCTC-3'. For the quantitative PCR analysis, PCR was performed in a $50 \mu 1$ reaction mixture that contained 1X PCR buffer, $0.2 \mathrm{mmol} / 1$ deoxynucleoside triphosphates (dNTPs), $0.4 \mu \mathrm{l}$ mol/l each primer, $50 \mathrm{ng}$ genomic DNA and 1 unit Tag DNA polymerase (Takara Biotechnology Co., Ltd., Dalian, China). PCR reactions were performed under the following conditions: Denaturation at $95^{\circ} \mathrm{C}$ for $4 \mathrm{~min}$, followed by 40 thermal cycles, each composed of $95^{\circ} \mathrm{C}$ for $30 \mathrm{sec}, 58^{\circ} \mathrm{C}$ for $30 \mathrm{sec}$ and $72^{\circ} \mathrm{C}$ for $45 \mathrm{sec}$. The PCR products were sequenced using an ABI3130 DNA analyzer (Applied Biosystems; Thermo Fisher Scientific, Inc., Waltham, MA, USA), according to the manufacturer's protocols. The results were compared with the NCBI reference sequence, NG_032652.1, to identify nucleotide substitutions.

Analysis of the conservation of amino acid residues. The amino acid sequences of human SKIV $2 L$ protein were aligned with those in the homologous proteins from eight eukaryotic species, including Pan troglodytes, Macaca mulatta, Felis catus, Mus musculus, Takifugu rubripes, Danio rerio, Drosophila melanogaster and Xenopus tropicalis.

The amino acid sequences for the proteins were collected from the ENSEMBL database at http:/www.ensembl.org/index. html. Sorting Intolerant From Tolerant (SIFT; http://sift.jcvi.org/) and Polymorphism Phenotyping v2 (PolyPhen-2; http://genetics. bwh.harvard.edu/pph2/index.shtml) databases were used to predict whether an amino acid substitution affected the function of the protein.

\section{Results}

Proband description. The proband was Han Chinese, was born at 35 weeks of gestational age with a birth weight of $1,480 \mathrm{~g}$ ( $<3$ rd centile) and was the first child of non-consanguineous parents; the mother had previously experienced three spontaneous abortions. Following birth, he was placed in an infant incubator and received artificial feeding. He reached a weight of 2,200 $\mathrm{g}$ at 4 weeks, and then suffered from watery diarrhea (10 times a day) and fever. The diarrhea was treated using protein hydrolysate, lactose-free formulas and rehydration, however, the diarrhea returned when the patient was fed with premature formula or normal infant formula. The diarrhea was described as watery, mucoid and occasionally bloody, and occurred between 5-10 times a day.

At 3 months of age, the patient suffered from an incarcerated oblique inguinal hernia and underwent surgical treatment with herniotomy and inguinal hernia repair. At 3 days-post surgery, he developed a severe lower respiratory tract infection and pleural effusion. Ascites and liver cirrhosis were also recorded in the course of the disease. At the age of 17 months old, he was hospitalized in our department (the Department of Gastroenterology, Nanjing Children's Hospital Affiliated to Nanjing Medical University, Nanjing, China) due to intractable diarrhea, severe protein energy malnutrition and failure to thrive. His physical examination demonstrated the following: Body weight, $6,500 \mathrm{~g}$ ( $<3$ rd centile); height, $71 \mathrm{~cm}$ ( $<3 \mathrm{rd}$ centile); poorly pigmented wooly hair; facial dysmorphism, including squared forehead, prominent cheeks, a broad nasal root and hypertelorism (Fig. 1). The liver was palpable $4 \mathrm{~cm}$ under the right costal margins. No mental retardation was noted. Conventional light microscopic analysis of the hair shaft revealed trichorrhexis nodosa (Fig. 1).

Laboratory investigations were performed to evaluate the proband's chronic diarrhea and failure to thrive, which found the following: Stool microscopy revealed 7-8 leukocytes/high power field and no parasites; stool cultures were negative for enteropathogens. The patient's complete blood count, liver enzymes, renal function tests and lipid profile were all within the normal range. Low immunoglobulin $\mathrm{G}, \mathrm{M}, \mathrm{C} 3$ and $\mathrm{C} 4$ levels were noted, and $\mathrm{T}$ cell immune functions showed a profile of high $\mathrm{CD}^{+}$lymphocytes, low $\mathrm{CD} 8^{+}$lymphocytes, high $\mathrm{CD}^{+} / \mathrm{CD}^{+}$cells and low natural killer cells. Metabolic evaluations, including tandem mass spectrometry, urine-blood amino acids and serum ammonia, were normal. The serum levels of $\alpha$-1-antitrypsin and ceruloplasmin were normal. Viral markers, including cytomegalovirus and hepatitis $\mathrm{A}, \mathrm{B}$ and $\mathrm{C}$, were all negative. Abdominal ultrasonography revealed hepatomegaly and cirrhosis.

Identification of a candidate mutation and genetic analysis. A length of $\sim 4.2 \mathrm{~kb}$ of the coding regions and the adjacent noncoding regions of the $S K I V 2 L$ gene (all exons) of the proband were captured, sequenced and analyzed in the present study. The sequence depth for each exon of the SKIV $2 L$ gene is shown in Fig. 2A, and the median depth across 28 exons was $68 \mathrm{x}$. The successful detection coverage included $100 \%$ of the SKIV $2 L$ gene exons. The sequencing identified two candidate variants, a 

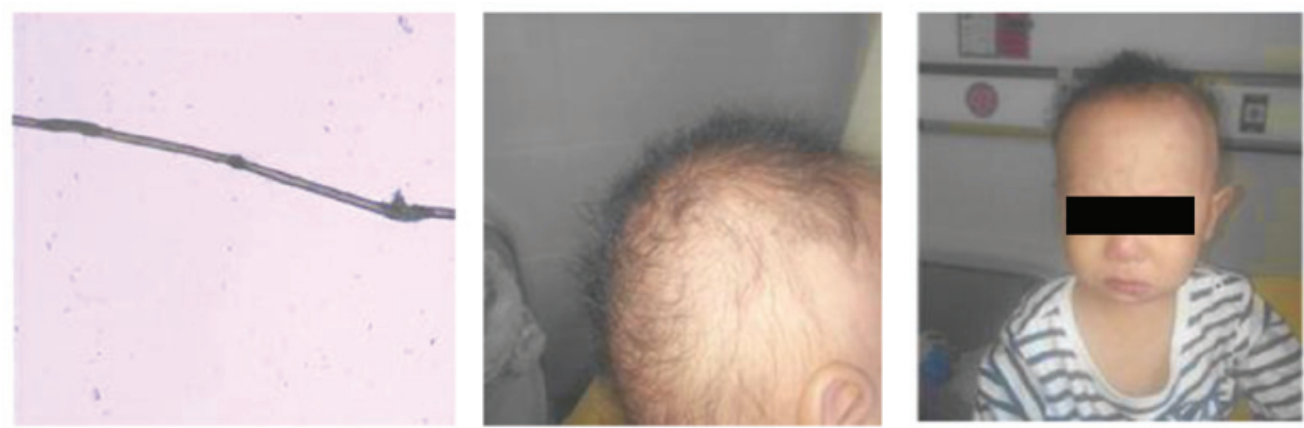

Figure 1. Physiological examination. The image on the left is from the microscopic hair examination, showing trichorrhexis nodosa. The images in the centre and right show the woolly hair and abnormal facial expression observations, respectively.

A

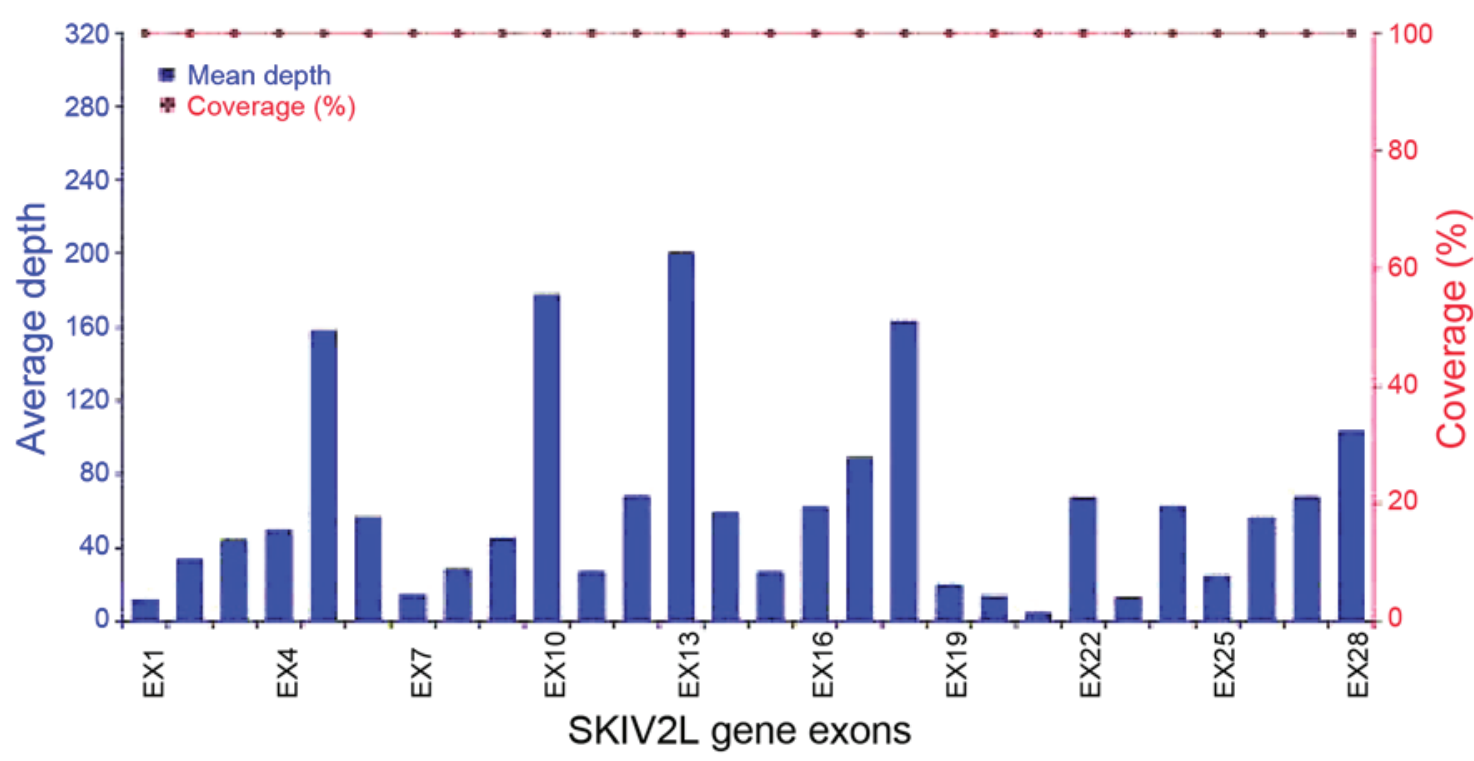

B

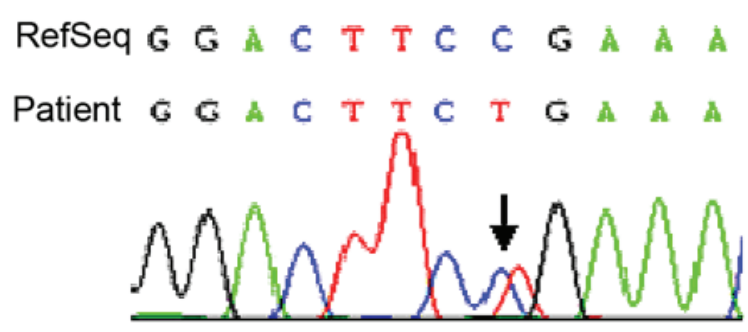

[c. $1120 \mathrm{C}>$ T. p. $374 \mathrm{R}>\mathrm{X}$ ]
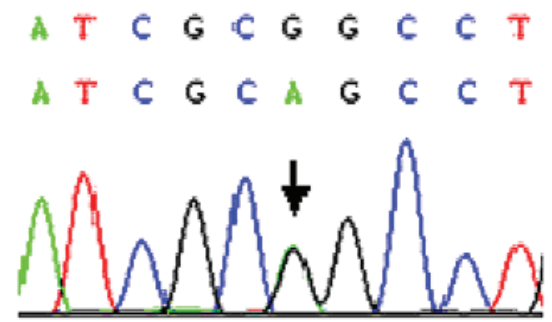

[c. $1891 \mathrm{G}>$ A. p. $631 \mathrm{G}>\mathrm{S}$ ]

Figure 2. Identification of $S K I V 2 L$ mutation depth and sequence coverage for all 28 exons (EX) in the $S K I V 2 L$ gene, determined via sequencing analysis of the $S K I V 2 L$ mutation. (A) Mean depth and coverage (\%). (B) DNA mutations; arrows indicate mutation sites. SKIV2L, superkiller viralicidic activity 2-like.

novel mutation (c.1891G>A) and a previously reported mutation (c.1120C>T), in the exon regions of the SKIV2L gene, which causes THES.

To confirm the single-nucleotide substitution mutations (c.1120C >T and c.1891G>A; SKIV2L; NM_600478), Sanger sequencing of the proband and his parents was performed. The two substitution variants in the SKIV $2 L$ gene were confirmed in the proband. Using the identical PCR primers and analysis with direct DNA sequencing, the c.1120C $>$ T variant in the $S K I V 2 L$ gene was detected in the DNA from his mother. The c.1891G>A variant in the SKIV2L gene was found in the

$\begin{array}{lll}\begin{array}{l}\text { Species } \\ \text { Human }\end{array} & \text { Gene } & \text { AA alignment } \\ \text { Mutated } & \text { ENST00000375394 } & 631 \text { QVLHMSELLNRGLGVHHSGILPIL } \\ \text { P.troglodytes } & \text { ENSPTRG00000017997 } & 631 \text { QVLHMSELLNRSLGVHHSGILPI } \\ \text { M.mulatta } & \text { ENSMMUG00000000736 } & 628 \text { QVLHMSELLNRGLGVHHSGILPI } \\ \text { F.catus } & \text { ENSFCAG00000004519 } & 631 \text { QVLHMSELLHRGLGVHHSGILPI } \\ \text { M.musculus } & \text { ENSMUSG00000040356 } & 628 \text { QVLHMSELLRRGLGVHHSGILPI } \\ \text { T.rubripes } & \text { ENSTRUG00000000966 } & 639 \text { QILLMRDLLKKGVAVHHSGILPI } \\ \text { D.rerio } & \text { ENSDARG00000062206 } & 640 \text { QILLMRDLLKRGIGVHHSGI } \\ \text { D.melanogaster } & \text { FBgn0039117 } & 581 \text { QVLVKDALERGIGVHHSGILPI } \\ \text { X.tropicalis } & \text { ENSXETG00000010932 } & 635 \text { QVLHMLDLLKRGIGIHHSGILPI }\end{array}$

Figure 3. Comparative alignment of the amino acid (AA) sequence of superkiller viralicidic activity 2-like. Amino acid conservation is indicated in bold. 
genomic DNA of his father, who was a carrier for the mutation (Fig. 2B).

The c.1120C $>\mathrm{T}$ variant resulted in the change of arginine to a termination codon at codon 374 of the SKIV2L protein (p.374R $>\mathrm{X}$ ), which was recorded in the SNP database (rs200818962). The novel SKIV2L mutation (c.1891G>A) caused a single glycine (Gly)-to-serine (Ser) change at amino acid 631 of the SKIV2L protein (p.631Gly>Ser). Phylogenetic analysis indicated that Gly at position 631 was highly conserved, even in the evolutionarily distant species of $P$. troglodytes, $M$. mulatta, F. catus, M. musculus, T. rubripes, D. rerio, D. melanogaster and $X$. tropicalis (Fig. 3). Furthermore, the Gly-to-Ser substitution at position 631 of the SKIV2L protein was predicted to affect the function of the protein, according to analyses using SIFT and PolyPhen-2. This mutation was predicted to be potentially damaging, with a score of 1.00 (probably damaging) by PolyPhen-2 and 0.00 (damaging) by SIFT. Taken together, this evidence indicated that $\mathrm{p} .631 \mathrm{Gly}>$ Ser was a pathogenic mutation associated with THES in the proband.

\section{Discussion}

Intractable severe diarrhea accompanied by phenotypic abnormalities and liver cirrhosis in infants is associated with THES. The prognosis of THES is poor, with $>25 \%$ of patients currently reported to succumb to mortality between the ages of 2-5 years, a proportion of which with early-onset cirrhosis. However, case reports have described improved survival rates, with long-term parenteral nutrition (PN)-dependency or PN weaning in certain cases. The patient described in the present study was considered to have THES, based on his intrauterine growth retardation, intractable diarrhea, facial dysmorphism, abnormal scalp hair shafts, trichorrhexis nodosa, immune disorders and liver cirrhosis.

Lee et al (11) isolated genomic clones and mapped the human homolog of the SKI2 gene to $6 \mathrm{p} 21$ using fluorescence in situ hybridization. Using genomic sequence analysis, Yang et al (12) determined that $S K I V 2 L$ is a polymorphic gene, which spans $11 \mathrm{~kb}$ and contains 28 exons (11). Fabre et al (10) sequenced the candidate gene, $S K I V 2 L$, in six unrelated patients with typical THES, who were known to be negative for mutations in the TTC37 gene, and identified homozygosity or compound heterozygosity for eight different mutations in all patients. To date, 12 mutations of the SKIV2L gene associated with THES have been found in the Human Gene Mutation Database (http://www. hgmd.cf.ac.uk/ac/gene.php?gene=SKIV2L). The present study provided the first report, to the best of our knowledge, of a novel missense mutation, c.1891G $>$ A, which resulted in a Gly-to-Ser change at amino acid 631 (p.631Gly $>$ Ser) in the encoded protein. The results of analyses using the two online software programs, PolyPhen-2 and SIFT, suggested that this mutation affected the structure and function of the protein. Furthermore, the novel c. $1891 \mathrm{G}>\mathrm{A}$ missense mutation was shown to be conserved in different species.

Although intractable diarrhea and the requirement for total PN are characteristic features of THES, certain infants have a milder phenotype. Barabino et al (13) reported cases of two patients on oral or enteral feeding at 8 and 7 years of age, respectively. Others have also reported cases of longer-term survival following weaning from PN $(1,14)$. The present study reported a case of the long-term survival of a patient with THES on oral feeding, suggesting a mild phenotype caused by heterozygous mutations, c.1891G $>$ A and c.1120C $>$ T, of the SKIV2L gene. Hepatic involvement in THES is reported to be variable, ranging from no abnormality to hemochromatosis and cirrhosis $(3,12,14)$. In the course of the 18 -month follow-up in the present study, the diarrhea persisted, however, there was no sign of progression of liver disease.

\section{Acknowledgements}

This study was sponsored by the Nanjing Municipal Health Bureau fund (grant no. YKK13132). The authors would like to thank the Joy Orient Translational Medicine Research Center Co., Ltd. for their assistance.

\section{References}

1. Fabre A, Breton A, Coste ME, Colomb V, Dubern B, Lachaux A, Lemale J, Mancini J, Marinier E, Martinez-Vinson C, et al: Syndromic (phenotypic) diarrhoea of infancy/tricho-hepato-enteric syndrome. Arch Dis Child 99: 35-38, 2014.

2. Fabre A, Martinez-Vinson C, Goulet $\mathrm{O}$ and Badens C: Syndromic diarrhea/Tricho-hepato-enteric syndrome. Orphanet J Rare Dis 8: 5, 2013.

3. Dweikat I, Sultan M, Maraqa N, Hindi T, Abu-Rmeileh S and Abu-Libdeh B: Tricho-hepato-enteric syndrome: A case of hemochromatosis with intractable diarrhea, dysmorphic features, and hair abnormality. Am J Med Genet A 143A: 581-583, 2007.

4. Hartley JL, Zachos NC, Dawood B, Donowitz M, Forman J, Pollitt RJ, Morgan NV, Tee L, Gissen P, Kahr WH, et al: Mutations in TTC37 cause trichohepatoenteric syndrome (phenotypic diarrhea of infancy). Gastroenterology 138: 2388-2398, 2398. e1-e2, 2010.

5. Goulet OJ, Brousse N, Canioni D, Walker-Smith JA, Schmitz J and Phillips AD: Syndrome of intractable diarrhoea with persistent villous atrophy in early childhood: A clinicopathological survey of 47 cases. J Pediatr Gastroenterol Nutr 26: 151-161, 1998.

6. Stankler L, Lloyd D, Pollitt RJ, Gray ES, Thom H and Russell G: Unexplained diarrhoea and failure to thrive in 2 siblings with unusual facies and abnormal scalp hair shafts: A new syndrome. Arch Dis Child 57: 212-216, 1982.

7. Verloes A,Lombet J,Lambert Y, Hubert AF, Deprez M, Fridman V, Gosseye S, Rigo J and Sokal E: Tricho-hepato-enteric syndrome: Further delineation of a distinct syndrome with neonatal hemochromatosis phenotype, intractable diarrhea, and hair anomalies. Am J Med Genet 68: 391-395, 1997.

8. Landers MC and Schroeder TL: Intractable diarrhea of infancy with facial dysmorphism, trichorrhexis nodosa, and cirrhosis. Pediatr Dermatol 20: 432-435, 2003.

9. Fabre A, Charroux B, Martinez-Vinson C, Roquelaure B, Odul E, Sayar E, Smith H, Colomb V, Andre N, Hugot JP, et al: SKIV2L mutations cause syndromic diarrhea, or trichohepatoenteric syndrome. Am J Hum Genet 90: 689-692, 2012.

10. Fabre A, Martinez-Vinson C, Roquelaure B, Missirian C, André N, Breton A, Lachaux A, Odul E, Colomb V, Lemale J, et al: Novel mutations in TTC37 associated with tricho-hepato-enteric syndrome. Hum Mutat 32: 277-281, 2011.

11. Lee SG, Lee I, Park SH, Kang C and Song K: Identification and characterization of a human cDNA homologous to yeast SKI2. Genomics 25: 660-666, 1995.

12. Yang Z, Shen L, Dangel AW, Wu LC and Yu CY: Four ubiquitously expressed genes, RD (D6S45)-SKI2W (SKIV2L)-DOM3Z-RP1 (D6S60E), are present between complement component genes factor B and C4 in the class III region of the HLA. Genomics 53: 338-347, 1998.

13. Barabino AV, Torrente F, Castellano E, Erba D, Calvi A and Gandullia P: 'Syndromic diarrhea' may have better outcome than previously reported. J Pediatr 144: 553-554, 2004.

14. Egritas O, Dalgic B and Onder M: Tricho-hepato-enteric syndrome presenting with mild colitis. Eur J Pediatr 168: 933-935, 2009. 